\title{
Expression and clinical significance of cyclooxygenase-2 and microRNA-143 in osteosarcoma
}

\author{
YONGCHAO FANG, ZHIQIANG ZHANG, QIANG WANG and JIANNING ZHAO \\ Department of Orthopedic Surgery, Jinling Hospital, Southern Medical University, Nanjing, Jiangsu 210002, P.R. China
}

Received July 1, 2014; Accepted March 5, 2015

DOI: $10.3892 /$ etm.2015.2420

\begin{abstract}
The aim of the present study was to investigate the expression profiles of cyclooxygenase (COX)-2 and microRNA (miRNA)-143 in the tumor tissue and blood samples of patients with osteosarcoma, and their involvement in the disease pathogenesis. Tumor tissue and blood samples were obtained from 46 patients with osteosarcoma (stages I-III). Reverse transcription-quantitative polymerase chain reaction (RT-qPCR) and western blot analyses were performed to detect the mRNA and protein expression of COX-2, respectively, in these samples. The expression of miRNA-143 in the tumor tissue and blood samples was assessed using RT-qPCR. The results showed that, compared with the normal control subjects, the mRNA and protein expression levels of COX-2 in the tumor tissue and blood samples of patients with osteosarcoma were increased. Among the patients with osteosarcoma, increases in the COX-2 mRNA and protein levels were observed with progressing disease severity (from stage I to stage III), suggesting the involvement of COX-2 in the disease pathogenesis. By contrast, the expression of miRNA-143 decreased as the disease progressed, which was the opposite trend to the COX-2 expression, indicating that miRNA-143 and COX-2 may play different roles in the disease pathogenesis. In conclusion, COX-2 expression in the tumor tissue and blood samples of patients with osteosarcoma increases significantly along with the degree of tumor malignancy, and this is accompanied by a decreased expression of miRNA-143; therefore, a negative correlation between COX-2 and miRNA-143 may exist in the progression of osteosarcoma.
\end{abstract}

\section{Introduction}

Osteosarcoma is a highly malignant primary tumor occurring in the bone and associated tissues, characterized by a bone-like tissue consisting of tumor cells (1). As the disease progresses, osteosarcoma can progressively harm the health

Correspondence to: Dr Jianning Zhao, Department of Orthopedic Surgery, Jinling Hospital, Southern Medical University, 305 Zhongshan East Road, Nanjing, Jiangsu 210002, P.R. China E-mail: zjndoctor@163.com

Key words: osteosarcoma, cyclooxygenase-2, microRNA-143 of the patients by causing lump formation, organ dysfunction and fractures (2-4). In the late stages, the majority of patients would develop metastases, resulting in systemic failure. At present, the exact mechanism of osteosarcoma pathogenesis is unclear. Certain studies have suggested that osteosarcoma can be induced by other types of cancer, as well as environmental stimuli to bone cells, such as toxins, viruses and radiation (5-7). In addition, disturbed hormone levels can increase the incidence of osteosarcoma, particularly in teenagers with excess hormone secretion (8).

A recent study found that cyclooxygenase (COX)-2 expression gradually increased in the development of osteosarcoma, suggesting its involvement in the progression of the disease (9). COX-2 has also been shown to play an important role in the pathogenesis of several other diseases, including gastric, pancreatic and bladder cancers, which makes COX-2 a promising biomarker in disease diagnosis (10-12). Additionally, microRNA (miRNA)-143 has been shown to influence the expression of COX-2 and affect disease progression; however, the expression of COX-2 in osteosarcoma, particularly concerning its association with miRNA-143, has not yet been fully elucidated.

The aim of the present study was to examine the expression profiles of COX-2 and miRNA-143 in tumor tissues and blood samples from patients with osteosarcoma and to discuss the association between the expression profiles and the disease severity. The findings could provide novel insights into the early diagnosis and clinical treatment of osteosarcoma.

\section{Materials and methods}

Patients and tissue samples. A total of 46 patients diagnosed with osteosarcoma who had been admitted to Jinling Hospital (Southern Medical University, Nanjing, China) between December 2011 and December 2013 were enrolled in the present study, including 22 stage I patients, 18 stage II patients and 6 stage III patients (Table I). In addition, 26 normal subjects were used as controls. A total of 20 osteosarcoma samples were obtained from the patients ( 9 from stage I patients, 8 from stage II patients and 3 from stage III patients), with adjacent tissues used as negative controls (Table II). Prior written and informed consent was obtained from every patient and the study was approved by the Ethics Review Board of Jinling Hospital. 
Table I. Basic patient information.

\begin{tabular}{lccccc}
\hline Group & Total (n) & Men (n) & Women (n) & Age range (years) & Mean age (years) \\
\hline Control & 26 & 16 & 10 & $10-28$ & 18.8 \\
Osteosarcoma I & 22 & 16 & 6 & $12-26$ & 18.6 \\
Osteosarcoma II & 18 & 12 & 6 & $11-27$ & 19.8 \\
Osteosarcoma III & 6 & 1 & 5 & $15-23$ & 18.1 \\
\hline
\end{tabular}

Table II. Information for osteosarcoma samples.

\begin{tabular}{lccccc}
\hline Group & Total (n) & Men (n) & Women (n) & Age range (years) & Mean age (years) \\
\hline Osteosarcoma I & 9 & 6 & 3 & $13-25$ & 17.6 \\
Osteosarcoma II & 8 & 2 & 6 & $11-20$ & 16.4 \\
Osteosarcoma III & 3 & 2 & 1 & $15-20$ & 17.3 \\
\hline
\end{tabular}

Reverse transcription-quantitative polymerase chain reaction $(R T-q P C R)$. Total RNA was extracted with TRIzol ${ }^{\circledR}$ reagent (Invitrogen Life Technologies, Carlsbad, CA, USA). The miRcute miRNA cDNA First Strand Synthesis kit (Tiangen Biotech Co., Ltd., Beijing, China) was used to perform the RT according to the manufacturer's instructions. qPCR was then performed with the miRcute miRNA qPCR detection kit (SYBR Green) (Tiangen Biotech Co., Ltd.). The primer sets used were synthesized by TIANGEN Biotech Co., Ltd., Beijing, China (Table III). The amplification conditions were as follows: $95^{\circ} \mathrm{C}$ for $5 \mathrm{~min}$; then $95^{\circ} \mathrm{C}$ for $50 \mathrm{sec}$ and $60^{\circ} \mathrm{C}$ for $30 \mathrm{sec}$, for 40 cycles. $\beta$-actin and U6 served as the internal controls for COX-2 and miRNA-143, respectively. The relative expression levels were calculated using the $2^{-\Delta \Delta \mathrm{Ct}}$ method.

Western blot analysis. Proteins were extracted from the tumor tissue and blood samples. A total of $20 \mu \mathrm{g}$ protein was subjected to $10 \%$ sodium dodecyl sulfate-polyacrylamide gel electrophoresis and transferred onto a polyvinylidene difluoride membrane. The membrane was incubated with rabbit anti-human anti-COX-2 polyclonal antibody $(1: 1,000$ dilution; cat. no. ab15191; Abcam, Boston, MA, USA) and rabbit anti-human anti- $\beta$-actin polyclonal antibody $(1: 5,000$ dilution; cat. no. ab8227; Abcam), respectively, at $4^{\circ} \mathrm{C}$ overnight. Horseradish peroxidase-conjugated goat anti-rabbit immunoglobulin G (1:3,000 dilution; cat. no. ab6721; Abcam) was then added and the membrane was incubated at room temperature for another hour. Coloration was performed using the Electrochemiluminescence Western Blot Substrate kit (Abcam), and the relative intensity was analyzed by Image $\mathrm{Lab}^{\mathrm{TM}}$ version 3.0 software (Bio-Rad Laboratories, Hercules, CA, USA).

Statistical analysis. Data are expressed as the mean \pm standard deviation. The SPSS software package (version 18.0; SPSS Inc., Chicago, IL, USA) was used for statistical analysis. Normality tests and one-way analysis of variance were performed for the comparison. $\mathrm{P}<0.05$ was considered to indicate a statistically significant difference.

\section{Results}

Expression of COX-2 in tumor tissues from patients with osteosarcoma. To investigate the mRNA and protein expression levels of COX-2 in osteosarcoma tumor tissue, RT-qPCR and western blot analysis were performed, respectively, in normal control subjects and patients with stages I-III osteosarcoma. Results from the RT-qPCR demonstrated that the mRNA expression levels of COX-2 in patients with osteosarcoma were significantly higher than those in the normal control subjects $(\mathrm{P}<0.05$; Fig. 1A). Furthermore, the mRNA expression level of COX-2 in patients with stage III osteosarcoma was significantly higher than that in patients with stage II osteosarcoma, which was significantly higher than that in patients with stage I osteosarcoma, indicating that the COX-2 mRNA expression level was increasing along with the disease severity. In addition, western blot analysis showed that the protein expression levels of COX-2 exhibited similar trends to COX-2 mRNA (Fig. 1B). These results suggested that the expression of COX-2 increased as the disease progressed, implying the involvement of COX-2 in the disease pathogenesis.

Expression of COX-2 in blood samples from patients with osteosarcoma. The mRNA and protein levels of COX-2 in blood samples from patients with osteosarcoma were measured by RT-qPCR and western blot analysis. The results showed that, compared with normal control subjects, the mRNA level of COX-2 was slightly elevated in the blood samples from patients with stage I osteosarcoma; however, the difference was not statistically significant $(\mathrm{P}>0.01$; Fig. 2A). In patients with stages II and III osteosarcoma, the COX-2 mRNA content in the blood samples was significantly higher than that in the normal controls $(\mathrm{P}<0.01)$, and the mRNA level was found to increase as the disease progressed (Fig. 2A). Similar results were observed with the western blot analysis of COX-2 protein expression in the blood samples from patients with osteosarcoma (Fig. 2B). These results indicated that the mRNA and protein levels of COX-2 in the 
Table III. Primer sets for the reverse transcription-quantitative polymerase chain reaction.

$\begin{array}{ll}\text { Primers } & \text { Sequences }\end{array}$

COX-2

\section{Forward}

Reverse

$\beta$-actin

Forward

Reverse

miRNA-143

Forward

Reverse

U6

Foward

Reverse

\author{
5'-CAGCCATACAGCAAATCCTTG-3' \\ 5'-CAAATGTGATCTGGATGTCAAC-3'
}

5'-CACCAGGGCGTGATGGT-3'

5'-CTCAAACATGATCTGGGTCAT-3'

5'-TGTAGTTCGGAGTTAGTGTCGCGC-3'

5'-CCTACGATCGAA AACGACGCGAACG-3'

5'-GTTTTGTAGTTTTTGGAGTTAGTGTTGTGT-3'

5'-CTCAACCTACAATCA AAAACAACACAAACA-3'

COX, cyclooxygenase; miRNA, microRNA.

A

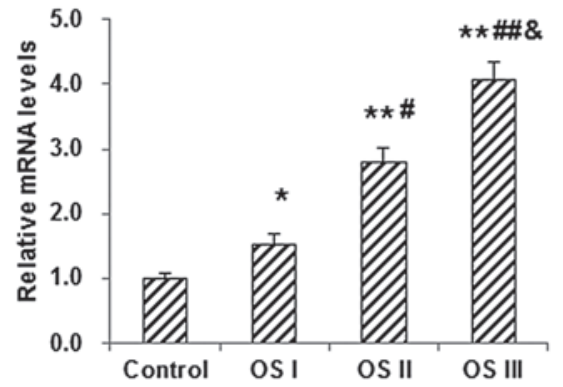

B
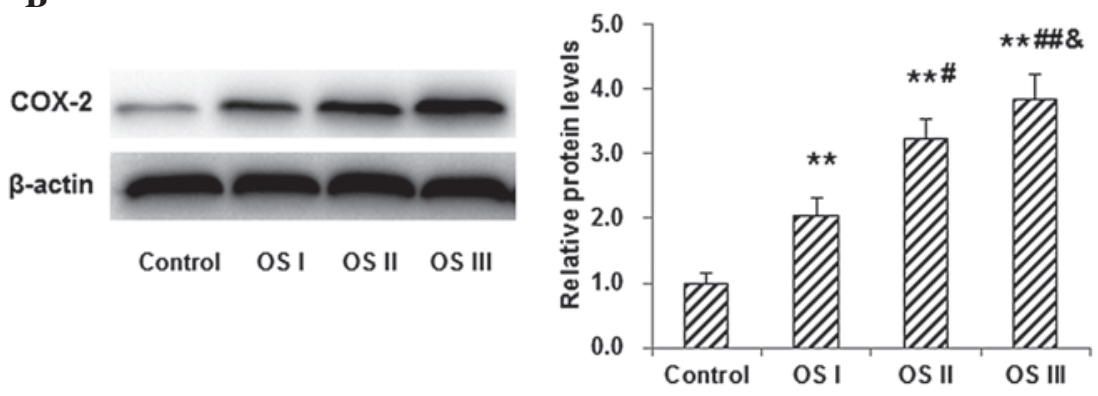

Figure 1. mRNA and protein expression of COX-2 in osteosarcoma tumor tissue. (A) mRNA expression levels in tumor tissue from normal control subjects and patients with stages I-III osteosarcoma were detected by the reverse transcription-quantitative polymerase chain reaction. (B) Protein expression levels in tumor tissue from normal control subjects and patients with osteosarcoma stages I-III were detected by western blot analysis. ${ }^{*}<0.05$ and ${ }^{* *} \mathrm{P}<0.01$ compared with the normal control; ${ }^{\#} \mathrm{P}<0.05$ and ${ }^{\# \#} \mathrm{P}<0.01$ compared with stage I osteosarcoma; ${ }^{\star} \mathrm{P}<0.05$ compared with stage II osteosarcoma. COX, cyclooxygenase; OS I, osteosarcoma stage I.
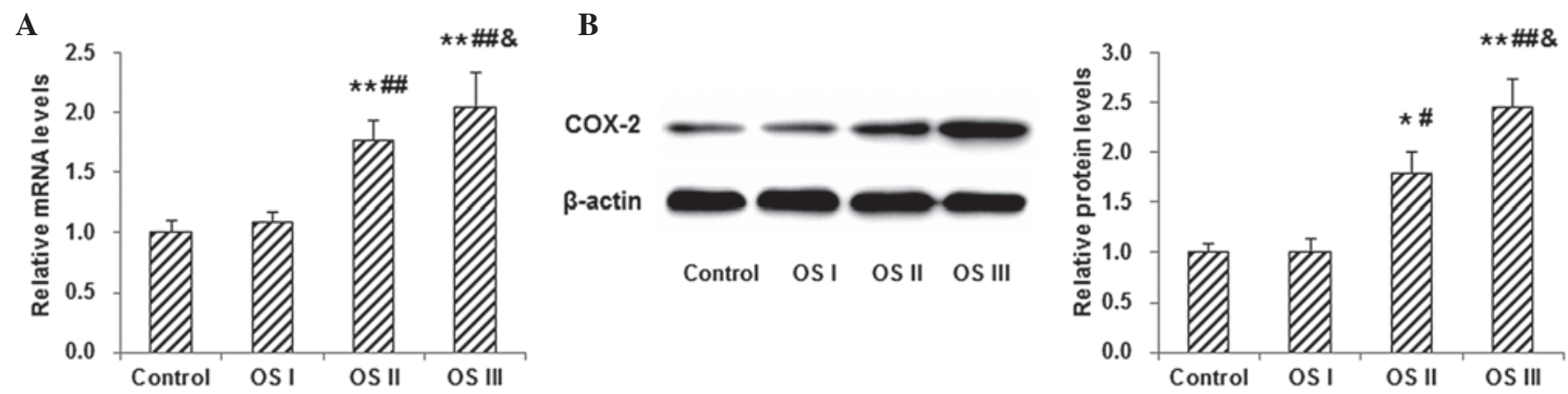

Figure 2. mRNA and protein expression of COX-2 in osteosarcoma blood samples. (A) mRNA expression levels in blood samples from normal control subjects and patients with stages I-III osteosarcoma were detected by the reverse transcription-quantitative polymerase chain reaction. (B) Protein expression levels in blood samples from normal control subjects and patients with osteosarcoma stages I-III were detected by western blot analysis. ${ }^{*} \mathrm{P}<0.05$ and ${ }^{* *} \mathrm{P}<0.01$ compared with the normal control; ${ }^{~} \mathrm{P}<0.05$ and ${ }^{\# \#} \mathrm{P}<0.01$ compared with stage I osteosarcoma; ${ }^{\circledR} \mathrm{P}<0.05$ compared with stage II osteosarcoma. COX, cyclooxygenase; OS I, osteosarcoma stage I.

blood were increased in patients with osteosarcoma, despite the fact that no significant difference was measured between patients with early-stage osteosarcoma and normal control subjects.
Expression of miRNA-143 in tumor tissue and blood samples from patients with osteosarcoma. To evaluate the role of miRNA-143 in osteosarcoma pathogenesis, its expression in osteosarcoma tumor tissue and blood samples was detected by 
A

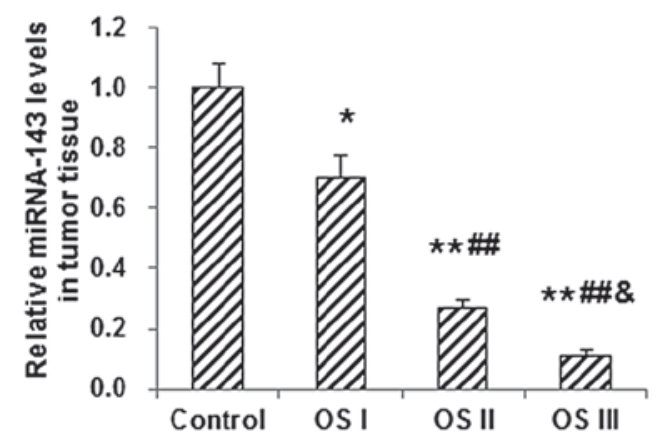

B

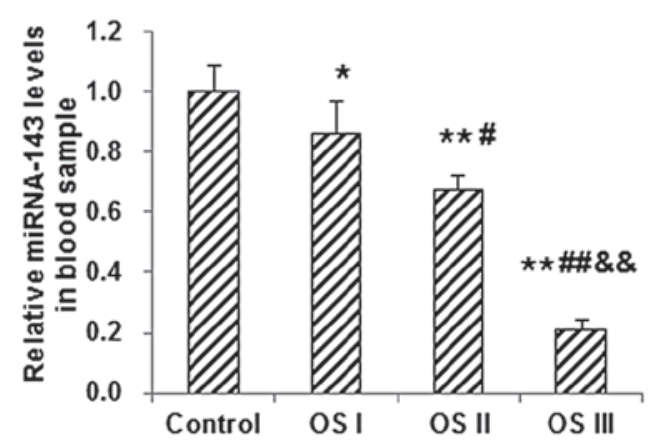

Figure 3. Expression of miRNA-143 in the tumor tissue and blood samples of patients with osteosarcoma. Levels of miRNA-143 in the (A) tumor tissue and (B) blood samples from normal control subjects and patients with stages I-III osteosarcoma were detected by the reverse transcription-quantitative polymerase chain reaction. ${ }^{*} \mathrm{P}<0.05$ and ${ }^{* *} \mathrm{P}<0.01$ compared with the control; ${ }^{\#} \mathrm{P}<0.05$ and ${ }^{\# \#} \mathrm{P}<0.01$ compared with stage I osteosarcoma; ${ }^{\&} \mathrm{P}<0.05$ and ${ }^{\& \&} \mathrm{P}<0.01$ compared with stage II osteosarcoma. miRNA, microRNA; OS I, osteosarcoma stage I.

RT-qPCR. The results showed that the level of miRNA-143 in osteosarcoma tumor tissue was significantly decreased in patients with stages I-III osteosarcoma, as compared with the level in the normal control subjects $(\mathrm{P}<0.05$; Fig. 3A). Among those patients with osteosarcoma, the miRNA-143 level in the tumor tissue declined along with the disease severity, i.e. the miRNA-143 levels in the tumor tissue from patients with osteosarcoma at stage III were significantly higher than those in tissue from patients at stages I-II, and the miRNA-143 level in stage II osteosarcoma was significantly higher than that in stage I osteosarcoma ( $\mathrm{P}<0.05$; Fig. 3A). Similar results were found with the measurement of miRNA-143 in the blood samples from patients with stages I-III osteosarcoma (Fig. 3B). These results indicated that the expression of miRNA-143 notably decreased along with the increasing severity of the osteosarcoma, which is the opposite trend to the COX-2 expression, suggesting that miRNA-143 and COX-2 may play different roles in the disease pathogenesis.

\section{Discussion}

Osteosarcoma is a highly malignant primary bone tumor, with bone-like tissue formed by tumor cells (1). As one of the early symptoms of osteosarcoma, patients experience intermittent pain, even prior to tumor formation. This intermittent pain can gradually transform into persistent, severe pain, particularly at night. Osteosarcoma can be caused by other types of cancer, including Li-Fraumeni syndrome (resulting from a p53 mutation), hereditary retinoblastoma (RB) and associated diseases caused by an RB mutation (13). Other internal or external factors can also contribute to the disease development $(6,14)$. In the present study, the pathogenesis of osteosarcoma, with regard to the expression of COX-2 and miRNA-143 and the association between them, was investigated.

COX-2 is the rate-limiting enzyme for the synthesis of prostaglandins, which are critically important for inflammation (15). Recent studies have indicated that COX-2 is also closely associated with the progression of various types of tumor $(16,17)$. The current results showed that the mRNA and protein expression levels of COX-2 became markedly upregulated as the disease severity increased. These results suggested that COX-2 may be involved in the development of osteosarcoma. The blood expression level of COX-2 may reflect the degree of malignancy and metastasis of osteosarcoma cells, since tumor metastasis largely relies on blood and tissue fluid flow. The present results showed that the mRNA and protein expression levels of COX-2 in the blood gradually increased from osteosarcoma stage I to stage III; however, the difference in the blood COX-2 expression level between the normal controls and patients with stage I osteosarcoma did not reach statistical significance. This may be due to the fact that a limited number of cells migrate in the blood circulation from the newly formed osteosarcoma tumor, and some of these would be phagocytosed by monocytes in the blood (18). COX-2 expression in the blood may, therefore, not be a perfect predictor for tumor metastasis, particularly for stage I osteosarcoma.

The present results demonstrated that the COX-2 expression levels in osteosarcoma tumor tissue increased along with the disease severity, from the normal control to osteosarcoma stages I, II and III, while the expression of miRNA-143 sequentially decreased as the disease progressed. We hypothesized that the upregulation of COX-2 may have been associated with the downregulation of miRNA-143 in the disease progression. miRNA-143 may therefore negatively regulate COX-2, which could be an important mechanism for chronic inflammation and the development of cancer.

The expression tendency of miRNA-143 in the blood was similar to that of COX-2 in the tumor tissues; it was, however, notable that, unlike COX-2, the difference in blood miRNA-143 expression between the normal controls and the patients with stage I osteosarcoma was statistically significant, suggesting that the blood miRNA-143 level may be a promising early predictor and diagnostic marker for osteosarcoma. Evidently, several potential questions remain. The regulatory mechanisms of miRNA-143, for example, are unclear, and there are also other factors that would affect COX-2 expression; therefore, further studies regarding the molecular mechanism and function of miRNA-143 in osteosarcoma are required.

In conclusion, the results of the present study showed that the mRNA and protein expression levels of COX-2 increased in the tumor tissue and blood samples from patients with osteosarcoma along with the disease severity. In addition, the 
expression of miRNA-143 decreased in the tumor tissue and blood samples from patients with osteosarcoma as the disease progressed. The opposite trends of COX-2 and miRNA-143 in the progression of osteosarcoma indicated that they played different roles in the disease pathogenesis. The current findings provided novel insights into the early diagnosis and clinical treatment of osteosarcoma.

\section{Acknowledgements}

The authors would like to thank Dr Sujia Wu, Dr Xinguang Zhou and Dr Tao Yuan from Jinling Hospital, Southern Medical University for their suggestions and assistance in the study design, sample and data collection, statistical analysis and manuscript preparation.

\section{References}

1. Lei P, Xie J, Wang L, Yang X, Dai Z and Hu Y: microRNA-145 inhibits osteosarcoma cell proliferation and invasion by targeting ROCK1. Mol Med Rep 10: 155-160, 2014.

2. Clark JC, Dass CR and Choong PF: A review of clinical and molecular prognostic factors in osteosarcoma. J Cancer Res Clin Oncol 134: 281-297, 2008.

3. Kansara M and Thomas DM: Molecular pathogenesis of osteosarcoma. DNA Cell Biol 26: 1-18, 2007.

4. Cai ZD and Li GD: Ezrin protein: Novel hot point of research on mechanism of neoplasm metastasis. Zhonghua Zhong Liu Za Zhi 6: 322-325, 2005 (In Chinese).

5. Gorlick R and Khanna C: Osteosarcoma. J Bone Miner Res 25: 683-691, 2010.
6. Ottaviani G and Jaffe N: The etiology of osteosarcoma. Cancer Treat Res 152: 15-32, 2009.

7. Kansara M, Leong HS, Lin DM, et al: Immune response to RB1-regulated senescence limits radiation-induced osteosarcoma formation. J Clin Invest 123: 5351-5360, 2013.

8. Zhou X, Jing J, Peng J, et al: Expression and clinical significance of galectin-3 in osteosarcoma. Gene 546: 403-407, 2014.

9. Wu X, Cai M, Ji F and Lou LM: The impact of COX-2 on invasion of osteosarcoma cell and its mechanism of regulation. Cancer Cell Int 14: 27, 2014.

10. Wu XL, Cheng B, Li PY, et al: MicroRNA-143 suppresses gastric cancer cell growth and induces apoptosis by targeting COX-2. World J Gastroenterol 19: 7758-7765, 2013.

11. Pham H, Rodriguez CE, Donald GW, et al: miR-143 decreases COX-2 mRNA stability and expression in pancreatic cancer cells. Biochem Biophys Res Commun 439: 6-11, 2013.

12. Song T, Zhang X, Wang C, et al: Expression of miR-143 reduces growth and migration of human bladder carcinoma cells by targeting cyclooxygenase-2. Asian Pac J Cancer Prev 12: 929-933, 2011.

13. Yamasaki L: Role of RB tumor suppressor in cancer. Cancer Treat Res 115: 209-239, 2003.

14. Jaffe N: Osteosarcoma. Pediatr Rev 12: 333-343, 1991.

15. Alhouayek M and Muccioli GG: COX-2-derived endocannabinoid metabolites as novel inflammatory mediators. Trends Pharmacol Sci 35: 284-292, 2014.

16. Wang L, Wang Z, Li J., et al NFATc1 activation promotes the invasion of U251 human glioblastoma multiforme cells through COX-2. Int J Mol Med: Mar 4, 2015 (Epub ahead of print). doi: $10.3892 /$ ijmm.

17. Wu K, Fukuda K, Xing F, et al: Roles of the cyclooxygenase 2-matrix metalloproteinase 1 pathway in brain metastasis of breast cancer. J Biol Chem: Feb 17, 2015 (Epub ahead of print). pii: jbc. M114.602185.

18. Toujas L, Delcros J G, Diez E, et al: Human monocyte-derived macrophages and dendritic cells are comparably effective in vitro in presenting HLA class I-restricted exogenous peptides. Immunol 91: 635-642, 1997. 